\title{
BMJ Open Healthcare-related cost of oropharyngeal dysphagia and its complications pneumonia and malnutrition after stroke: a systematic review
}

\author{
Sergio Marin (D) , ${ }^{1,2}$ Mateu Serra-Prat (1) , 3,4 Omar Ortega (ib) , ${ }^{1,4}$ Pere Clavé (1) ${ }^{1,4}$
}

To cite: Marin S, Serra-

Prat M, Ortega 0 , et al. Healthcare-related cost of oropharyngeal dysphagia and its complications pneumonia and malnutrition after stroke: a systematic review. BMJ Open 2020;10:e031629. doi:10.1136/ bmjopen-2019-031629

- Prepublication history and additional material for this paper are available online. To view these files, please visit the journal online (http://dx.doi. org/10.1136/bmjopen-2019031629).

Received 16 May 2019 Revised 03 March 2020 Accepted 28 May 2020
Check for updates

(C) Author(s) (or their employer(s)) 2020. Re-use permitted under CC BY-NC. No commercial re-use. See rights and permissions. Published by BMJ.

For numbered affiliations see end of article.

Correspondence to

Dr Sergio Marin;

sergiomarinrubio@gmail.com

\section{ABSTRACT}

Objectives To assess the healthcare costs associated with poststroke oropharyngeal dysphagia (OD) and its complications (malnutrition, dehydration, pneumonia and death).

Design Systematic review following Preferred Reporting Items for Systematic Reviews and Meta-Analyses recommendations.

Data sources MEDLINE, Embase and the National Health Service Economic Evaluation Database were searched up to 31 December 2019.

Participants Patients with poststroke.

Primary outcome measures The costs associated to poststroke OD and its complications.

Data analysis Data were synthetised narratively, quality evaluation was done using an adaptation of Drummond's checklist and Grading of Recommendations Assessment, Development and Evaluation recommendations were used to assess strength of evidence.

Results A total of 166 articles were identified, of which 10 studies were included. The cost of OD during the hospitalisation was assessed in four studies. One prospective study showed an increase of US\$6589 for patients requiring tube feeding. Two retrospective studies found higher costs for those patients who developed OD, (US\$7329 vs US\$5939) among patients with haemorrhagic stroke transferred to inpatient rehabilitation and an increase of $€ 3000$ (US\$3950) and SFr14 000 (US\$15300) in hospitalisation costs. One study did not found OD as a predictor for total medical costs in the multivariate analysis. One retrospective study showed an increase of US\$4510 during the first year after stroke for those patients with $\mathrm{OD}$. For pneumonia, five retrospective studies showed an increase in hospitalisation costs after stroke of between US\$1456 and US\$27633. One prospective study showed an increase in hospitalisation costs during 6 months after stroke in patients at high malnutrition risk. Strength of evidence was considered moderate for $\mathrm{OD}$ and pneumonia and low for malnutrition.

Conclusions This systematic review shows moderate evidence towards higher costs for those patients who developed $\mathrm{OD}$ after stroke. The available literature is heterogeneous, and some important aspects have not been studied yet. Further studies are needed to define the specific cost of poststroke OD.

PROSPERO registration number CRD42018099977.
Strengths and limitations of this study

- This systematic review was performed using the Preferred Reporting Items for Systematic Reviews and Meta-Analyses recommendations.

- The bibliographic research considers MEDLINE, Embase and the National Health Service Economic Evaluation Database databases.

- A quality evaluation using an adaptation of the Drummond's tool was performed.

- Strength of evidence was assessed using the Grading of Recommendations Assessment, Development and Evaluation methodology.

- The heterogeneity between the included studies and the variation on costs depending on the context precluded a quantitative data synthesis.

\section{INTRODUCTION}

Oropharyngeal dysphagia (OD) is a common condition in patients with poststroke, as a result of the brain injuries suffered. ${ }^{1}$ Incidence is high $(37 \%-78 \%)$ in the acute phase ${ }^{2}$ and, while improvements can be observed in many patients during the first weeks after stroke, OD persists as a chronic condition in nearly $50 \%$ and complications arise. ${ }^{3}$ The latest editions of the International Classification of Diseases (ICD) and Related Health Problems promoted by WHO ICD-9 and ICD-10 classifies poststroke OD with specific codes I-69.391. ${ }^{4}$

OD can have a high impact on the general health of affected patients and can produce two main types of complications in patients with poststroke: (1) those caused by impaired efficacy of swallow, present in $25 \%-75 \%$ of patients, which leads to malnutrition and dehydration $^{5}$ and (2) impaired safety of swallow which leads to tracheobronchial aspiration that may cause pneumonia in $50 \%$ of cases. ${ }^{6-10}$ Both OD and aspiration are highly prevalent conditions in patients with stroke. ${ }^{26}$ A recent study on patients with 
stroke has shown a prevalence of OD of $45.06 \%$ on admission, and that OD after stroke was an independent risk factor for prolonged hospital stay and institutionalisation after discharge. Moreover, this study has shown that OD was independently associated with poorer functional capacity and increased mortality 3 months after stroke. ${ }^{6} \mathrm{~A}$ significant increase in length of stay and a poor prognosis has also been observed by other authors in patients with poststroke OD. ${ }^{11}$ The impact of all these complications on the costs of poststroke OD is still unknown.

The state-of-the-art of OD management in patients with poststroke aims for early detection, with swallowing being assessed in the first hours after stroke diagnosis ${ }^{12}$ in order to prevent the potential complications of OD. Application of specific explorations in the acute setting, therapy development aimed at compensating deficient mechanisms related to this pathology and the recovery of swallowing function in these patients in the long term are key aspects of the management of this condition. ${ }^{13}$ Poststroke OD is an underdiagnosed and undertreated condition and the most appropriate care is not available for most patients. Significant reductions in rates of pneumonia (9.0\% vs $2.8 \%)$ and mortality $(7.4 \%$ vs $4.2 \%)$ have been demonstrated through screening and basic OD treatment application and is reflected in antibiotic expenditure with significant savings of around $50 \% .^{14}$ The treatment paradigm for poststroke OD is changing from compensatory strategies, which aim to compensate deficient mechanisms related to OD by using fluid adaptation and postural changes, to the recovery of swallowing function with the promotion of brain plasticity with neurorehabilitation techniques such as transcutaneous or intrapharyngeal electrical stimulation, repetitive transcranial magnetic stimulation and transcranial direct current stimulation. These neurorehabilitation strategies are still in the research phase but can already be perceived as an important progress in poststroke OD management. ${ }^{15}$

Costs related to poststroke OD comprise acute in-hospital and long-term sanitary costs, direct non-healthcare costs, indirect costs such as productivity losses and intangible costs. In the recent years, more data on the cost of poststroke OD has become available. However, no systematic literature review has been conducted as yet on this topic. The objective of this systematic review is to assess and summarise all the evidence on the cost related to OD and its complications (malnutrition, dehydration, aspiration pneumonia and death) in patients with poststroke. This study is a first step towards establishing the cost benefits of appropriate management of poststroke OD as an aspect to be taken into consideration by healthcare decision-makers.

\section{METHODS}

This systematic review was carried out using methodology Preferred Reporting Items for Systematic Reviews and Meta-Analyses. ${ }^{1617} 18$ In summary, a systematic review of studies related to the cost of OD and its complications (malnutrition, dehydration, aspiration pneumonia and death) in patients with stroke was performed. The main outcome of interest was the additional costs attributable to poststroke OD and its complications during the hospitalisation and follow-up after discharge. Task organisation in this systematic review including those processes performed by two or more authors (selection of studies, data extraction, quality assessment) is explained in the protocol of this systematic review.

\section{Patient and public involvement statement}

There was no public or patient involvement in the elaboration of this systematic review.

\section{Search strategy}

We searched Medline, Embase and the National Health Service Economic Evaluation Database (NHS EED) up until 31 December 2019. The references of the studies included were further revised to identify possible additional eligible studies. Used search terms are available in the protocol of this systematic review and in the online supplementary appendix. ${ }^{17} 18$

\section{Eligibility criteria}

Studies were included if they were cost studies, studies that provide information on costs in adult ( $>17$ years) patients with stroke with OD and/or its complications or economic evaluation studies in which the cost of the disease was estimated. Studies were excluded if they were not related to OD or if they refer to oesophageal dysphagia or OD caused by causes other than stroke. Full-text assessments were done in order to reject those ones not fulfilling all selection criteria. Two independent reviewers participated in this process. In case of disagreement over one or more studies, a third reviewer revised the study and a final consensus was made. A posterior task was carried out to identify possible duplicated information between the articles.

\section{Data presentation and summary measures}

Data were reported in its original format using tables and narrative. A narrative method was used to synthesise this evidence. Results are presented according to the following order: (1) costs related to poststroke OD, (2) costs related to OD complications in the following order: (A) aspiration, (B) pneumonia, (C) malnutrition, (D) dehydration and (E) death. A synthesis of studies separating acute and long-term costs was performed. Whenever feasible, data on cost adjusted for the stroke severity (according to National Institutes of Health Stroke (NIHSS) or Canadian scale) or other confounding factors were considered. Information was presented following recommendations of the Centre for Reviews and Dissemination. ${ }^{19}$ Results were also discussed as those assessing cost during the hospitalisation compared with cost after discharge and long-term follow-up.

\section{Quality evaluation and strength of the evidence}

In this study, we used an adaptation of Drummond's checklist in order to assess the risk of bias and the reporting 
Table 1 Main design characteristics

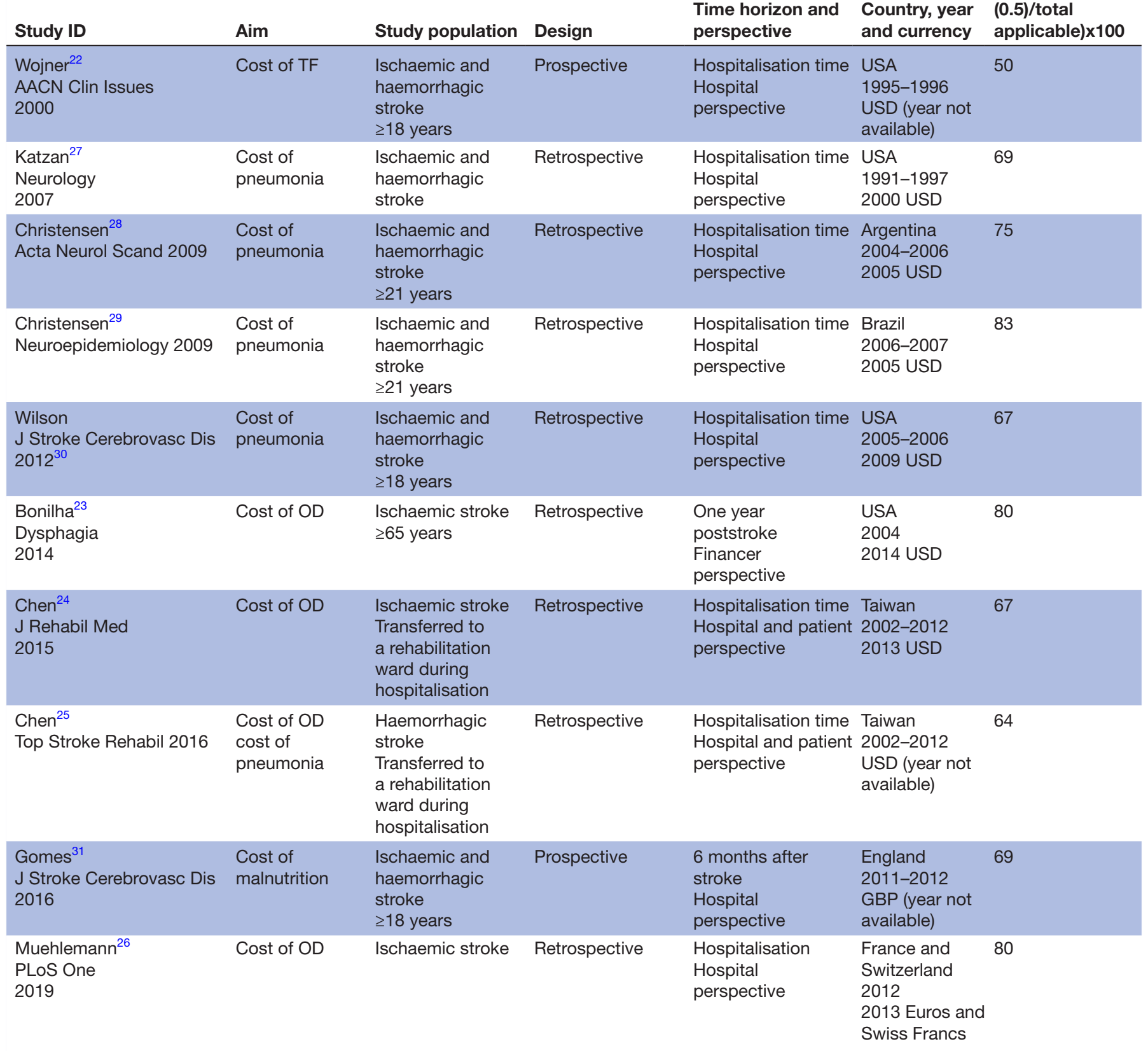

${ }^{*}$ Quality assessment: a higher score indicates a lower risk of bias.

$\mathrm{OD}$, oropharyngeal dysphagia; TF, tube feeding.

quality for each study only using the points in the checklist that were applicable to cost studies. ${ }^{20}$ Each of these points was rated as: yes, no, partly, not available or not applicable. Two independent reviewers participated in this process. A global score presented as a percentage was calculated for each study dividing the total number of points rated as 'yes' ('partly' counted as 0.5 ) between the total points applicable for each study. No study was excluded from this review based on risk of bias results. A higher score indicates a lower risk of bias. Quality assessment of the studies in this systematic review is presented in table 1 and expanded in the online supplementary appendix. In addition, we rated the quality of evidence across studies as high, moderate, low or very low using Grading of Recommendations Assessment, Development and Evaluation methodology. ${ }^{21}$ The hypothesis of this systematic review is that OD and its main complications are related to high costs in patients with poststroke. If a study demonstrated significantly higher costs for patients with poststroke who developed OD than for those who did not, higher costs 


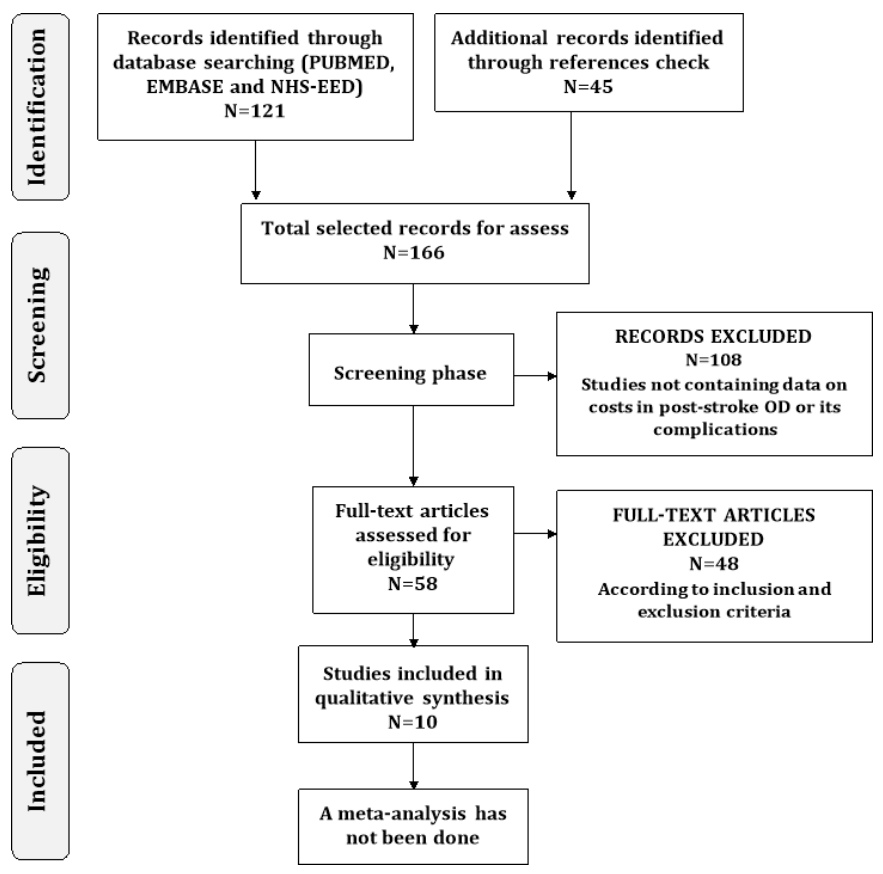

Figure 1 Selection process flow diagram. NHS EED, National Health Service Economic Evaluation Database; OD, oropharyngeal dysphagia.

for those who develop a complication related to OD or an effect of OD or its complications on total costs of stroke, the study was qualified as a 'positive study'.

\section{RESULTS}

In the data base search, 121 articles were identified using the search terms (67 through Medline using PubMed, 12 through Embase using Ovid and 42 through NHS EED) and 45 articles were identified through reference check. A total of 166 studies were assessed in the selection phase. After screening both title and abstract of these articles, 108 articles were excluded because they did not provide information on poststroke OD costs or those of its complications or at least minimal relevant information on this aspect. A second evaluation phase was carried out with the 58 remaining studies. After this second evaluation phase, 48 articles were excluded because they did not meet the criteria for inclusion (4 were duplicated articles, 38 did not provide information on costs and 6 did not refer to OD) and 10 articles were included in this systematic review (figure 1). Included study data and features were summed up and presented in both a narrative presentation and evidence (tables 1-4). A great heterogeneity regarding the economic design among studies was found (mainly among study perspective, type of included costs and follow-up time). Results were, therefore, not comparable and studies were evaluated separately.

\section{Costs of OD after stroke}

Five studies have assessed the cost of OD in patients with stroke from different perspectives and using different methodologies. These five studies had a longitudinal design. Studies' sample size ranged from 171 to 68334 participants and the mean age of participants ranged from 61.3 to 78.1 years. ${ }^{22-26}$ Two studies were performed in the USA, ${ }^{22} 23$ two were performed in Taiwan, ${ }^{24}{ }^{25}$ and one was performed in France and Switzerland. ${ }^{26}$ The US studies and the study performed in France and Switzerland were cost analysis studies ${ }^{22} 2326$ and the Taiwanese studies were cost prediction studies. ${ }^{24}{ }^{25}$ Incidence of OD ranged from $4.2 \%$ to $65.9 \%$. Screening for OD on admission was performed only in the study by Wojner and Alexandrov. ${ }^{22}$ One study analysed hospitalisation costs in patients depending on tube feeding (TF) from a hospital perspective. ${ }^{22}$ One study analysed hospital care, nursing home, care provider, home health, outpatient and durable medical equipment costs during the first year after ischaemic stroke and from the perspective of Medicare. ${ }^{23}$ Two studies analysed hospital costs including diagnoses, ward, laboratory, X-rays, therapeutic and surgical procedures, blood/plasma, anaesthesia, special materials, TF, rehabilitation, drugs, dispensing and injection services, haemodialysis and psychiatric treatment for patients transferred to a rehabilitation ward after ischaemic ${ }^{24}$ and haemorrhagic ${ }^{25}$ stroke from the hospital and patient perspectives. One study analysed the cost of hospital stay from the hospital perspective. ${ }^{26}$

Wojner and Alexandrov found significantly higher costs during the hospitalisation for those patients who depended on a TF. Mean hospitalisation costs were US\$12 $538 \pm$ US $\$ 6247$ for those patients who depended on a TF and US $\$ 5949 \pm$ US $\$ 3428$ for those who did not $(\mathrm{p}<0.0001)$, suggesting a non-adjusted effect of TF of approximately US $\$ 6300$. The study also showed that those patients depending on a TF were older, with a greater neurological impairment and had longer hospital stays. NIHSS score was found to be an independent risk factor for TF dependency. ${ }^{22}$ Bonilha et al found an increase of US $\$ 4510(p<0.0001)$ on the total medical costs during the first year after stroke for patients with ischaemic stroke who developed OD, controlling for age, comorbidities, ethnicity and time alive. Nevertheless, stroke severity was not shown as a significant independent predictor for the cost model.$^{23}$ Chen et al examined the predictors for total medical costs in first ischaemic stroke patients transferred to a rehabilitation ward in Taiwan. In this study, OD was significantly related with total medical costs during hospitalisation in the univariate analysis but not in the multivariate analysis. Significant predictors for total medical costs after multivariate analysis were: impaired consciousness, hypoalbuminaemia, fever, hypokalaemia and hyponatraemia. Mean total costs for dysphagia patients during the hospitalisation were $5134.5 \pm 3064$.6, and total medical costs for all patients (including those who already had dysphagia) were US $\$ 4606.8 \pm$ US $\$ 2926.1 .{ }^{24}$ Following the same methodology and perspectives, Chen et al examined the predictors for total medical costs in patients with a first haemorrhagic stroke event. This study shows that $\mathrm{OD}$ is related to a significant increase in the total medical costs with a beta coefficient of 1025.8 (95\% CI 


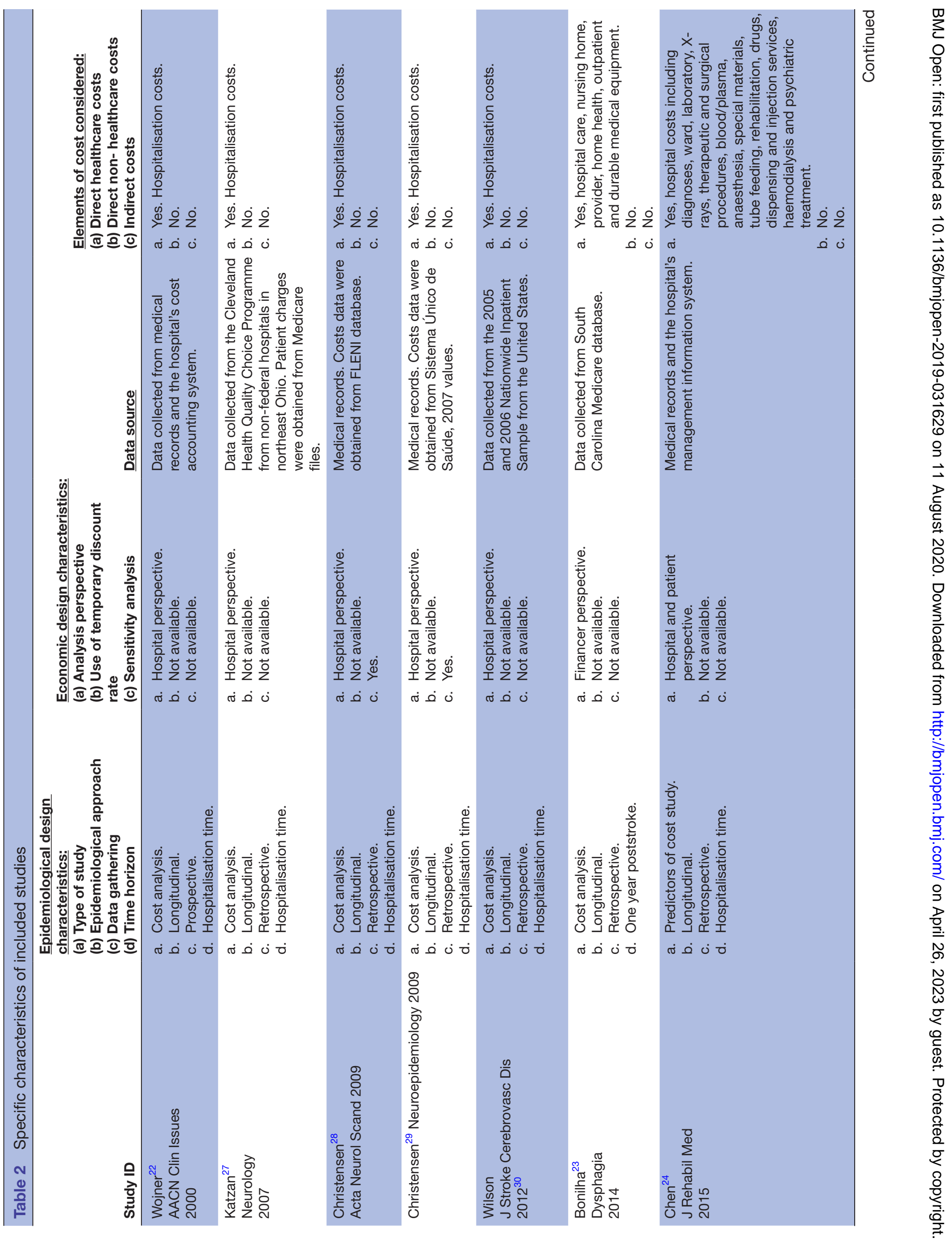




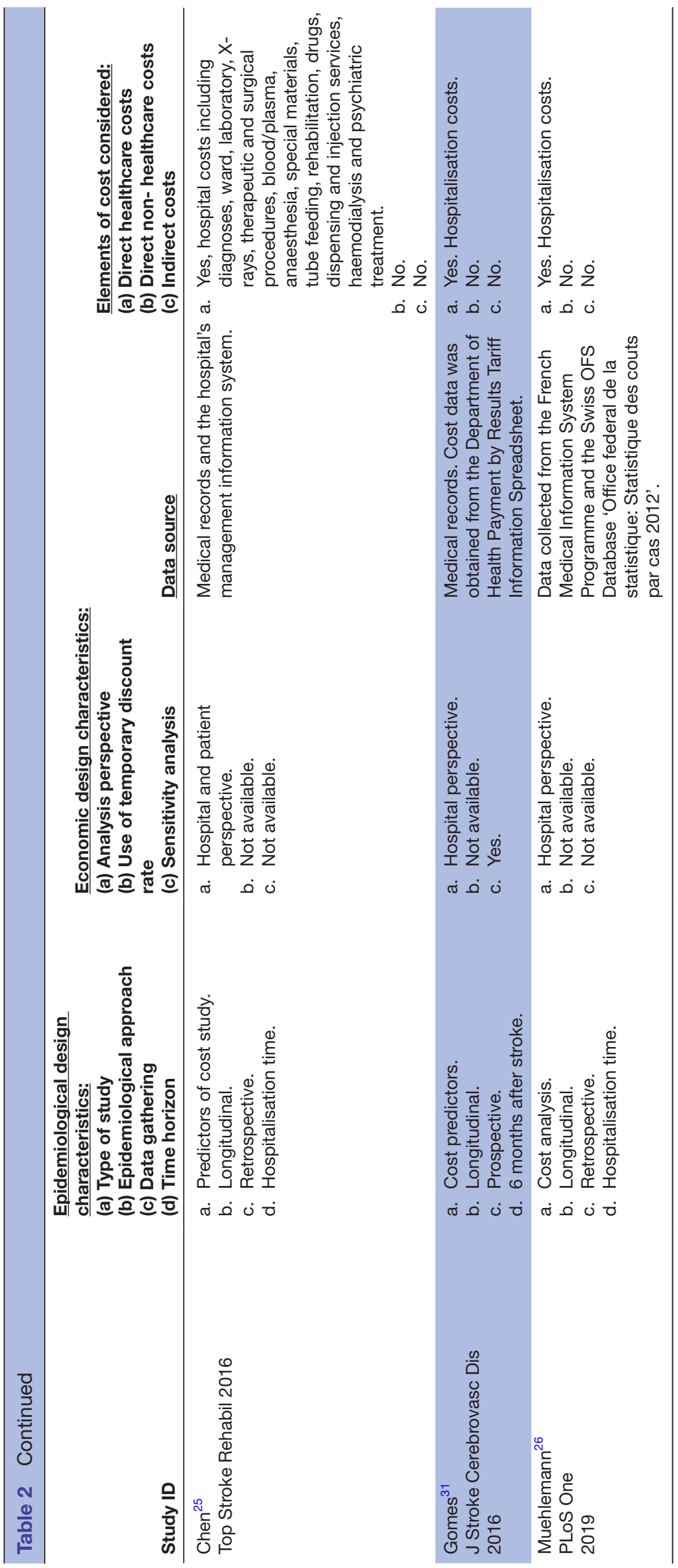

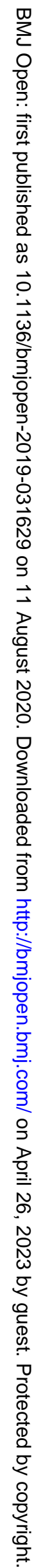




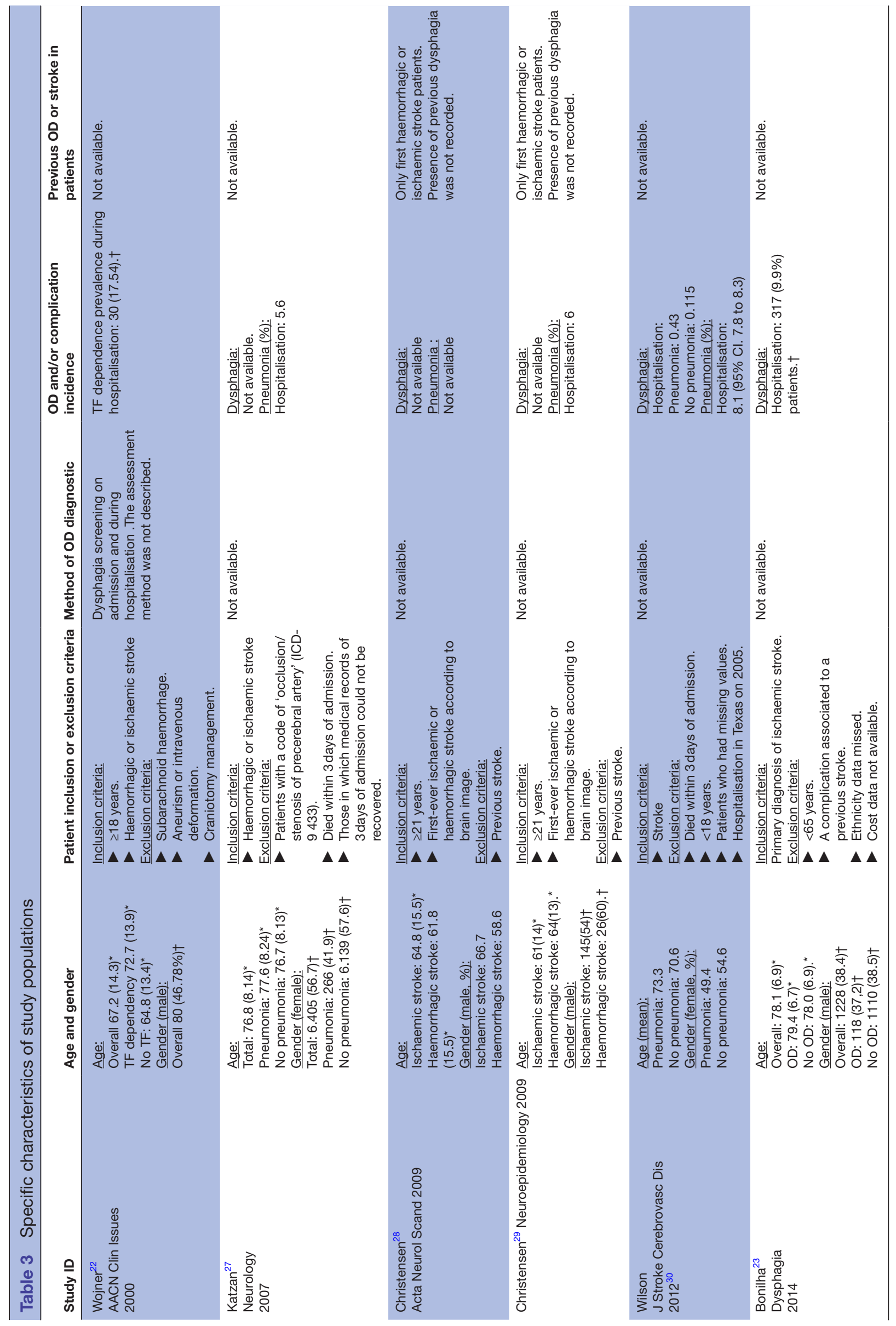




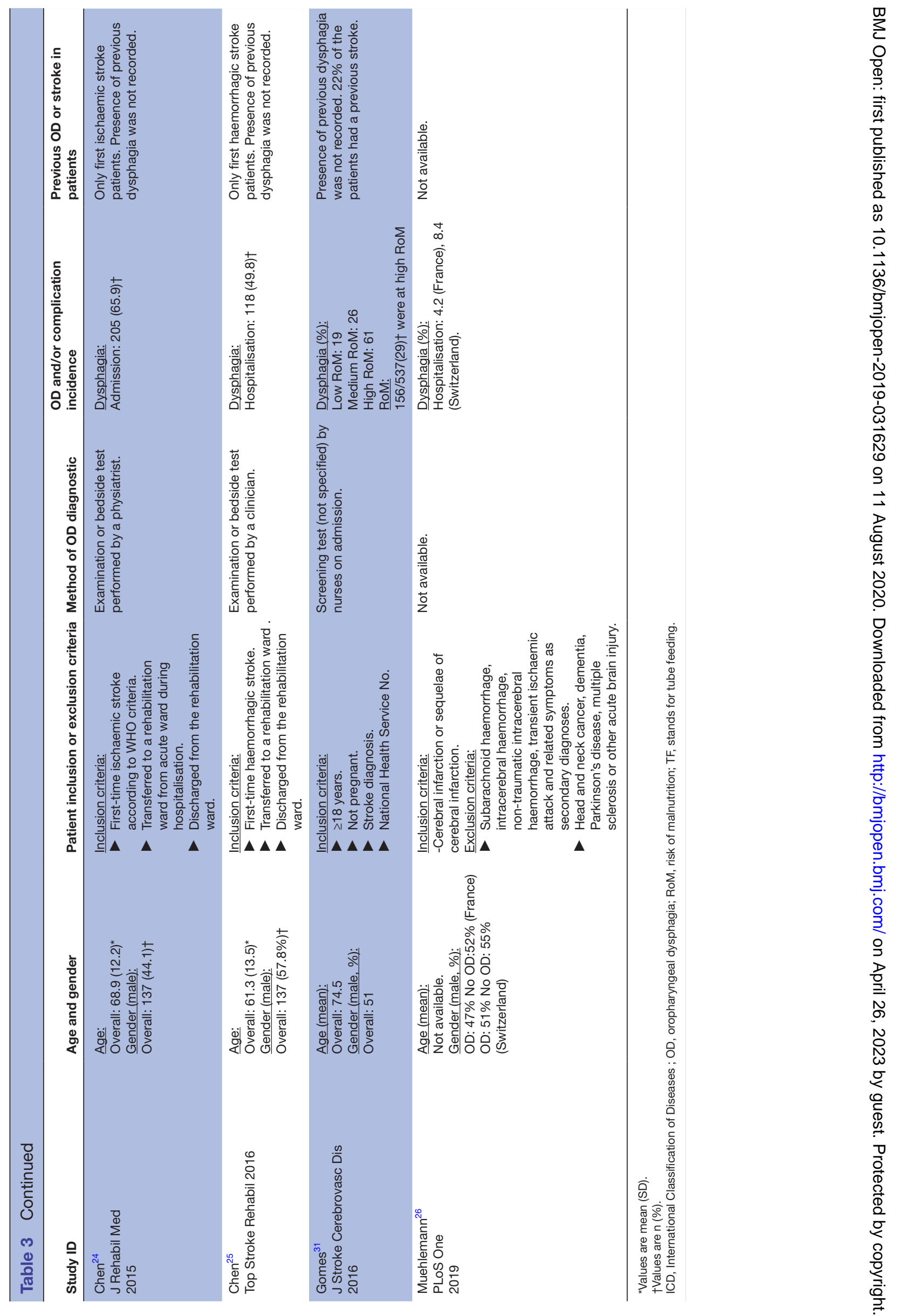


Table 4 Results of individual studies

\begin{tabular}{|c|c|c|c|c|c|c|}
\hline Study ID & Aim & $\begin{array}{l}\text { Sample } \\
\text { size }\end{array}$ & Crude incremental costs & $P$ value & $\begin{array}{l}\text { Adjusted } \\
\text { incremental } \\
\text { costs }\end{array}$ & $P$ value \\
\hline $\begin{array}{l}\text { Wojner } 22 \\
\text { AACN Clin Issues } \\
2000\end{array}$ & Cost of TF & 171 & $\begin{array}{l}\text { Mean cost for TF patients: } \\
\text { US } \$ 12538 \pm \text { US } \$ 6247 . \\
\text { Mean cost for } \\
\text { non-TF patients: } \\
\text { US } \$ 5949 \pm \text { US } \$ 3428=\end{array}$ & $<0.0001$ & - & - \\
\hline $\begin{array}{l}\text { Christense }^{28} \\
\text { Acta Neurol Scand } 2009\end{array}$ & $\begin{array}{l}\text { Cost of } \\
\text { pneumonia }\end{array}$ & 167 & $\begin{array}{l}\text { Ischaemic stroke: } \\
\text { US } \$ 36149 \\
\text { Haemorrhagic stroke: } \\
\text { US } \$ 16893\end{array}$ & $\begin{array}{l}=0.003 \\
=0.003\end{array}$ & - & - \\
\hline $\begin{array}{l}\text { Wilson } \\
\text { J Stroke Cerebrovasc Dis } \\
2012^{30}\end{array}$ & $\begin{array}{l}\text { Cost of } \\
\text { pneumonia }\end{array}$ & 183976 & US\$23102 & - & $\begin{array}{l}\text { US\$27 } 633(95 \% \\
\text { CI US\$27078 to } \\
\text { US\$27 988) }\end{array}$ & - \\
\hline $\begin{array}{l}\text { Chen }^{24} \\
\text { J Rehabil Med } \\
2015\end{array}$ & Cost of OD & 311 & $\begin{array}{l}\text { Mean cost for OD patients: } \\
\text { US } \$ 5134.5 \pm \text { US } \$ 3064.6 \\
\text { Mean patients cost: } \\
\text { US } \$ 4606.80 \pm \text { US } \$ 2926.1\end{array}$ & $<0.001$ & - & - \\
\hline $\begin{array}{l}\text { Chen }^{25} \\
\text { Top Stroke Rehabil } 2016\end{array}$ & $\begin{array}{l}\text { Cost of OD } \\
\text { Cost of } \\
\text { pneumonia }\end{array}$ & 237 & $\begin{array}{l}\text { Mean cost for OD patients: } \\
\text { US } \$ 7329.2 \pm \text { US } \$ 3977.2 \text {. } \\
\text { Mean cost for patients who } \\
\text { developed pneumonia: } \\
\text { US } \$ 9053.7 \pm U S \$ 5142.0 \text {. } \\
\text { Mean patients cost: } \\
\text { US } \$ 5939.5 \pm \text { US } \$ 3578.5 D\end{array}$ & $\begin{array}{l}<0.001 \\
<0.001\end{array}$ & - & - \\
\hline
\end{tabular}


Table 4 Continued

\begin{tabular}{|c|c|c|c|c|c|c|}
\hline Study ID & Aim & $\begin{array}{l}\text { Sample } \\
\text { size }\end{array}$ & Crude incremental costs & $P$ value & $\begin{array}{l}\text { Adjusted } \\
\text { incremental } \\
\text { costs }\end{array}$ & $P$ value \\
\hline $\begin{array}{l}\text { Gomes }^{31} \\
\text { J Stroke Cerebrovasc Dis } \\
2016\end{array}$ & $\begin{array}{l}\text { Cost of } \\
\text { malnutrition }\end{array}$ & 543 & $\begin{array}{l}\text { Median costs low-risk } \\
\text { patients: } 4920 \text { ( } £ 437-£ 38 \\
200) \\
\text { Median costs medium-risk } \\
\text { patients: } 6490 \text { ( } £ 1050-£ 19 \\
600) \\
\text { Median costs high-risk } \\
\text { patients: } 8720 \text { ( } £ 552-£ 31 \\
900)\end{array}$ & $<0.001$ & - & - \\
\hline $\begin{array}{l}\text { Muehlemann }{ }^{26} \\
\text { PLoS One } \\
2019\end{array}$ & Cost of OD & $\begin{array}{l}62297(F) \\
6037(S)\end{array}$ & $\begin{array}{l}2926 \text { euros (F) } \\
13959 \text { Swiss Francs (S) }\end{array}$ & - & - & - \\
\hline
\end{tabular}

F, France; OD, oropharyngeal dysphagia; R, range minimum-maximum; S, Switzerland; TF, tube feeding.

193.9 to $1857.8 ; \mathrm{p}<0.001)$. Total cost for OD patients was US $\$ 7329.2 \pm$ US $\$ 3977.2$ while total medical cost for all patients (including those who already had OD or pneumonia) was US\$5935.5 \pm US\$3578.5. ${ }^{25}$ Muehlemann et al found an incremental cost for patients who had OD of $€ 3000$ (approx. US $\$ 4300,2019$ USD) in France and SFr14000 (approx. US $\$ 16900,2019$ USD) in Switzerland. OD was associated with a significant increase in hospital costs during admission after adjusting for the presence of motor or/and sensory stroke complications $(p<0.0001) .{ }^{26}$

\section{Cost of poststroke safety of swallow complications: pneumonia}

We found five studies that evaluated the cost of pneumonia in patients with stroke, ${ }^{25}{ }^{27-30}$ one of which has been previously mentioned for providing information on the cost of OD after stroke. ${ }^{25}$ These five studies had a longitudinal, retrospective cost analysis design with sample sizes between 167 and 183976 participants with a mean age of $61.3-76.8$ years. ${ }^{25}{ }^{27-30}$ Two studies were performed in the USA, ${ }^{27} 30$ one in Taiwan, ${ }^{25}$ one in Argentina ${ }^{28}$ and one in Brazil. ${ }^{29}$ Presence of OD among analysed patients was reported in two studies. ${ }^{25}{ }^{30}$ Chem et al found that $49.8 \%$ of the assessed patients suffered OD during hospitalisation ${ }^{25}$ and Wilson et al found that OD was present in $42.9 \%$ of patients who developed pneumonia and in $11.5 \%$ of those who did not $(\mathrm{p}<0.0001){ }^{30}$ Pneumonia incidence ranged from $5.6 \%$ to $8.1 \%{ }^{25} 27-30$ The studies, except for the one performed by Chen and $\mathrm{Ke},{ }^{25}$ analysed hospitalisation costs after stroke from the hospital perspective. ${ }^{27-30}$

Chen and Ke showed that pneumonia was related to a significant increase in the total medical costs with a beta coefficient of 2330.1 (95\% CI 1339.5 to $3320.7 ; \mathrm{p}<0.001$ ). Total cost for patients who developed pneumonia was US $\$ 9053.7 \pm$ US $\$ 5142.0$ while total medical cost for all patients (including those who already had pneumonia) was US $\$ 5939.5 \pm \mathrm{US} \$ 3578.5 .^{25}$ Katzan et al showed an incremental cost for patients who developed pneumonia during the hospitalisation of US\$14863 (95\% CI 14436 to 15 236) adjusting for stroke severity, stroke patients' propensity for pneumonia and other factors associated with higher hospitalisation costs. Pneumonia was more commonly identified in those patients with a more severe stroke $(\mathrm{p}<0.001) .{ }^{27}$ Wilson found an additional adjusted cost of US\$27633 (95\% CI 27078 to 27 988) for a pneumonia episode during hospitalisation after stroke. Costs were adjusted for age, gender, hospital factors (teaching, rural, urban), admission from emergency department, illness severity, propensity for pneumonia and comorbidities. ${ }^{30}$ Christensen et al estimated that patients who developed pneumonia incurred significantly higher costs in both ischaemic (US\$36149; $\mathrm{p}=0.003$ ), and haemorrhagic stroke (US $\$ 16893 ; \mathrm{p}=0.003$ ) in Argentina. ${ }^{28}$ Finally, in the Christensen et al study performed in Sao Paulo, Brazil, an increase in hospitalisation cost for patients who developed pneumonia was observed for haemorrhagic stroke (US\$8485 vs US $\$ 3553 ; \mathrm{p}=0.015$ ) and for ischaemic stroke (US $\$ 4251$ vs US $\$ 1776 ; \mathrm{p}<0.001$ ). Development of pneumonia during admission was found to be a significant independent predictor of acute treatment costs in the multivariate analysis. An adjusted cost of pneumonia of US\$1456 was found when adjusting for all patient and treatment characteristics $(\mathrm{p}<0.001){ }^{29}$

\section{Cost of poststroke efficacy of swallow complications: malnutrition}

Gomes et al aimed to assess the validity of a nutritionscreening tool (Malnutrition Universal Screening Tool) to predict poor outcomes and hospitalisation costs in patients after a stroke episode. A total of 543 patients were enrolled in a longitudinal, prospective study. Costs were measured during 6 months after stroke from a hospital perspective. Mean age of participants was 74.5 years. The study was performed in London in two hyperacute stroke units and compared the hospitalisation costs according to risk of malnutrition. The study showed that an increase in malnutrition risk involved an increase in hospitalisation 
costs, from $£ 4920$ sterling (GBP) 2011 (approx. US $\$ 8780$, 2019 USD) in low risk patients to $£ 8720$ (approx. US $\$ 15$ 560,2019 USD) in high-risk patients $(\mathrm{p}<0.001)$. Risk of malnutrition was identified as an independent predictor for hospitalisation costs. Moreover, the study showed an association between high risk of malnutrition and inadequate swallow on hospital admission. ${ }^{31}$

\section{Synthesis of the study's findings \\ Short-term cost of OD and its complications during hospital admission}

In the case of patients admitted to a rehabilitation ward due to haemorrhagic stroke, the median cost for those who developed OD was US\$7329.2 while for the entire sample it was US $\$ 5939.5 .{ }^{25}$ In the case of ischaemic stroke patients admitted to a rehabilitation ward, OD was not identified as a predictor for the total medial costs in the multivariate analysis. An additional cost for those patients may be US $\$ 528$ per case. ${ }^{24}$ For patients who needed TF, an approximate increase of US\$6300 was observed. ${ }^{22}$ For patients hospitalised in France and Switzerland after stroke an increase of $€ 3000$ and SFr 14000 was observed. ${ }^{26}$ In the five studies reporting data on the cost of pneumonia after stroke, an adjusted effect of pneumonia on costs during hospitalisation after stroke was found to be between US\$1456 and US\$27633 depending on the setting and the design of the study. ${ }^{25-30}$

Long-term cost of OD and it's complications including post hospital discharge costs

We found only two studies assessing cost related to OD or it's complications beyond the first hospital stay due to acute stroke. The study performed by Bonilha et al found an increase of US $\$ 4510$ for those patients who developed OD during the first year after acute stroke. ${ }^{23}$ In malnutrition, Gomes et al showed an increase in all hospitalisation costs during the first 6 months after stroke. These costs increased from $£ 4920$ (approx.US\$8780, 2019 USD) in low risk of malnutrition patients to $£ 8720$ (approx. US $\$ 15$ 560,2019 USD) in high-risk patients. ${ }^{31}$

\section{Quality assessment}

Risk of bias was assessed for each study. Total scores for each study are available in table 1 and specific results for each study are available in the online supplementary appendix. Productivity losses secondary to the pathology were not assessed in any of the studies and quantities of consumed resources were only reported by Christensen et $a l$ and Muehlemann $e t a l$ and separately from their unit $\operatorname{costs}^{2628}$ Methods for the estimation of quantities and units costs were barely described in the studies, the studies by Katzan et al and Christensen $e t$ al were the only ones to completely include them. ${ }^{27-29}$ A sensitivity analysis was only offered in the studies by Christensen $e t$ al and Gomes et al. ${ }^{28} 2931$ The majority of data for these studies was retrospectively obtained from databases based on diagnosis codes. This can cause major information loss when calculating a disease cost as some conditions are underdiagnosed. We suspect that OD and/or pneumonia underdiagnosis could occur in some studies due to the absence of screenings for OD and low codification of these events in the databases.

\section{Confidence in cumulative evidence}

In the case of OD, one prospective and four retrospective studies were assessed. The risk of bias ranged from 50 to 80 . High consistency with a large and direct effect was found among the results of four studies. In the study performed by Chen et al, OD was significantly correlated with the total medical costs in univariate analysis but not in multivariate analysis so we have not qualified this study as a positive study. ${ }^{24}$ We consider the level of evidence regarding higher costs related to poststroke OD to be moderate. In the case of pneumonia, we also consider the level of evidence in favour of higher costs related to this complication to be moderate based on the five retrospective positive studies. The risk of bias punctuation ranged from 64 to 83. Despite the fact that the longitudinal design of the considered studies indicates moderate strength of scientific evidence, a high consistency with a large and direct effect was found among the results of the different studies. In the case of malnutrition, we only found one prospective study and the level of evidence was low.

\section{DISCUSSION}

This systematic review shows moderate quality evidence towards higher costs for those patients who developed OD or pneumonia after stroke. Acute in-hospital costs related to OD were analysed in five studies. Despite this, design and results were very different across studies and it is difficult to show definitive conclusions as a quantitative synthesis of the results cannot be made. The results of these economic studies do not match recent clinical studies that clearly show OD after stroke is an independent risk factor for prolonged hospital stay and institutionalisation after discharge; OD has been shown to be an independent risk factor for poorer functional capacity and increased mortality 3 months after stroke. ${ }^{6}$ A recent study performed in the USA also related OD to longer length of stay, higher inpatient costs and likelihood of being transferred to postacute care facility and inpatient mortality during hospitalisation. ${ }^{32}$ Monetisation of these poor clinical outcomes specifically caused by OD is urgent and would probably indicate specific incremental costs for OD. Regarding the costs of pneumonia, Christensen et alshowed, in Brazil and Argentina, an increase in hospitalisation costs for stroke patients who developed pneumonia of between US\$1456 and US\$36149 depending on the type of the stroke and the country. ${ }^{28}{ }^{29}$ These higher health costs more closely approximate what we estimate could be the increment in costs from OD and pneumonia from the clinical trials we are performing: US $\$ 27633$ to US $\$ 36149$ seem reasonable and can be translated to a 2019 USD value of US\$32500 to US $\$ 48000$. In the study performed by Gomes et al, an association between OD 
and malnutrition is observed on admission. However, this study did not directly assess OD costs or deglutition alterations and it cannot be directly extrapolated that malnutrition costs were a consequence of OD although we can assume that malnutrition in OD patients can also lead to higher $\operatorname{costs}^{31}$ It should be noted that the cost associated with complications related to OD accounted for a large part of the total costs of hospitalisation in the included studies and that it is possible that hospitalisation costs do not include all the costs attributable to these complications. Health economic studies on the cost of other complications of stroke are scarce. No article measuring aspiration, dehydration or mortality costs was found.

We believe that from our data we can estimate the cost of OD in the acute phase might be up to $€ 15000$ (approx. US\$16900) and the cost of an episode of aspiration pneumonia up to $€ 24000$ (approx. US\$27600). Our systematic review differs from a previously published systematic review because it focused on poststroke OD and explored the costs related to OD complications. This previous systematic review aimed to assess the influence of OD secondary to all aetiologies on length of stay and costs showed an increase of $40.36 \%$ in costs of patients with OD. A subgroup evaluation showed a higher and more variable length of stay of 4.73 days $(95 \%$ CI 2.7 to 7.2) for patients with stroke. ${ }^{33}$

Two important aspects in this field must be highlighted. First, poststroke OD is a condition for which effective interventions are available that may reduce long-term cost and related complications. Costs related to caring for poststroke OD patients have been little reported. Further research should be done to assess the poststroke OD economic and social burden to better understand and raise awareness about minimal care for this common and severe complication in patients with poststroke. It is becoming increasingly difficult to ignore the need for early screening and basic treatment in poststroke OD patients to reduce their mortality rate and improve their outcomes. Second, in most of the included studies, patients with poststroke were not screened for OD so only the most severe patients may have been diagnosed with OD. This fact could affect the prevalence of OD in these studies and the results of this systematic review and explain the underdiagnosis of OD in some of these studies compared with the literature. ${ }^{2}$ Furthermore, the high presence of false negatives could have led to a dilution of the effect. A future prospective study on costs related to poststroke OD in a sample of patients screened early for dysphagia could provide more accurate results.

We found only one study providing information on OD cost beyond the first admission due to acute stroke. However, we could not find any economic evaluation regarding other relevant complications of poststroke OD such as: (1) the need for institutionalisation after discharge, (2) loss of functional capacity, (3) costs related to home care of these patients, (4) and the related shortterm and long-term mortality and impaired quality of life. ${ }^{6}$ Furthermore, costs of patient care outside the acute hospital setting, social costs and non-sanitary costs have been barely studied. This systematic review shows the need for future high-quality studies to quantify the acute and chronic cost of poststroke OD and its specific complications.

\section{CONCLUSIONS}

This systematic review only partially fulfils the proposed objectives. The studies found were conducted in very different contexts and following very different approaches, limiting the analysis to a narrative explanation of what has been investigated to date. In addition, for those studies related to complications associated with OD, the relationship between OD and the complication was not clearly established. Despite this, this systematic review shows increased economic costs during hospitalisation and long-term follow-up in patients who developed poststroke OD or its complications. Future studies on OD after stroke on patients screened and detected early for OD will enable the long-term costs of OD and the true cost of its severe complications to be calculated. Taking into account the chronic nature of this condition, it is necessary to discover the real health and social costs associated with this pathology. In addition, due to the severe complications that patients with poststroke with OD present and their increased associated cost, it would be interesting to assess the cost-effectiveness of the current available treatments for these patients.

\section{Author affiliations}

${ }^{1}$ Gastrointestinal Physiology Laboratory, Universitat Autònoma de Barcelona, Hospital de Mataró, Consorci Sanitari del Maresme, Mataro, Catalunya, Spain ${ }^{2}$ Pharmacy Department, Hospital Universitari Germans Trias i Pujol, Badalona, Catalunya, Spain

${ }^{3}$ Research Unit, Hospital de Mataró, Consorci Sanitari del Maresme, Mataro, Catalunya, Spain

${ }^{4}$ Centro de Investigación Biomédica en Red de enfermedades hepáticas y digestivas (CIBERehd), Instituto de Salud Carlos III, CIBEREHD, Barcelona, Spain

Acknowledgements The authors thank Agustí Viladot, member of Mataró Hospital Library Service, for his assistance with literature search development and realization, Jane Lewis for reviewing the English and Maurice Driessen for reviewing the final version of this manuscript and advice on healthcare economics. This work has been conducted within the framework of a doctoral thesis in medicine from the Medicine Department of the Autonomous University of Barcelona.

Contributors SM is the guarantor. He drafted the first version of this manuscript He provided expertise on health economics. He contributed to the realisation of the introduction and the conclusions. He reviewed and extracted data of selected studies and contributed with Drummond's and GRADE application. He contributed with data synthesis. MS-P provided expertise on investigation methodology and health economics. He collaborated in writing the manuscript. He provided important references for the development of the methodology of this work. He reviewed and made contributions on the methodology of all the sections of this protocol. He reviewed the correct use of all the economic terms included in this manuscript. He reviewed and extracted data of selected studies and contributed with Drummond's and GRADE application. He contributed with data synthesis. He read and approval the final revision of this systematic review. 00 provided expertise on poststroke $\mathrm{OD}$ treatments and interventions. He collaborated in writing the manuscript. He contributed on the development of the data collection, quality assessment and data synthesis sections. He provided a critical revision of all the sections of this systematic review. He reviewed and extracted data of selected studies and contributed with Drummond's and GRADE application. He contributed with data synthesis. PC provided expertise on poststroke OD and the design of the study. 
He reviewed the correct use of all the medical terms included in this manuscript. He contributed on the study design, and the development and writing of the introduction, the methodology and the conclusions of the manuscript. He provided a critical revision of all the sections of this protocol. He contributed to the correct following of the recommendations proposed by PRISMA. He provided a critical revision of all the sections of this systematic review. He reviewed and extracted data of selected studies and contributed with Drummond's and GRADE application. He contributed with data synthesis. All authors provided a critical revision and read and approved the final revision of this systematic review. All authors contributed with the realisation of the protocol for this systematic review.

Funding This study is funded by an educational grant from Nutricia Advanced Medical Nutrition. This study is supported by Fundació Salut del Consorci Sanitari del Maresme, Ciberehd (in turn supported by the Instituto de Salud Carlos III, Barcelona, Spain) and Fundació de Recerca en Gastroenterologia (FUREGA).

Competing interests None declared.

Patient consent for publication Not required.

Ethics approval This systematic review is the first part of a research project. The full extent of this project includes (1) a systematic review on the cost of OD and its complications after stroke; (2) a systematic review on economic evaluations of interventions related to screening, diagnosis, management and treatment of OD after stroke; (3) a cost of illness study with one year follow up in patients with poststroke to assess the acute and chronic cost of $\mathrm{OD}$ and its complications; and (4) a cost-effectiveness study of compensatory versus active interventions aimed at recovering swallowing function in patients with poststroke.

Provenance and peer review Not commissioned; externally peer reviewed.

Data availability statement No additional data is available.

Open access This is an open access article distributed in accordance with the Creative Commons Attribution Non Commercial (CC BY-NC 4.0) license, which permits others to distribute, remix, adapt, build upon this work non-commercially, and license their derivative works on different terms, provided the original work is properly cited, appropriate credit is given, any changes made indicated, and the use is non-commercial. See: http://creativecommons.org/licenses/by-nc/4.0/.

\section{ORCID iDs}

Sergio Marin http://orcid.org/0000-0001-7709-6504

Mateu Serra-Prat http://orcid.org/0000-0002-6554-9913

Omar Ortega http://orcid.org/0000-0001-6178-3748

Pere Clavé http://orcid.org/0000-0002-0696-8560

\section{REFERENCES}

1 Rofes L, Arreola V, Romea M, et al. Pathophysiology of oropharyngeal dysphagia in the frail elderly. Neurogastroenterol Motil 2010;22:851-e230.

2 Martino R, Foley N, Bhogal S, et al. Dysphagia after stroke: incidence, diagnosis, and pulmonary complications. Stroke 2005;36:2756-63.

3 Mann G, Hankey GJ, Cameron D. Swallowing function after stroke: prognosis and prognostic factors at 6 months. Stroke 1999;30:744-8.

$4 \mathrm{WHO}$, International classification of disease [online], 2016. Available: http://www.who.int/ classifications/icd/en

5 Ortega O, Cabré M, Clavé P. Oropharyngeal Dysphagia: Aetiology \& Effects of Ageing. Journal of GHR 2014;3:1049-54.

6 Rofes L, Muriana D, Palomeras E, et al. Prevalence, risk factors and complications of oropharyngeal dysphagia in stroke patients: a cohort study. Neurogastroenterol Motil 2018;23:e13338.

7 Ortega O, Martín A, Clavé P. Diagnosis and management of oropharyngeal dysphagia among older persons, state of the art. $J$ Am Med Dir Assoc 2017;18:576-82.

8 Cabre M, Serra-Prat M, Palomera E, et al. Prevalence and prognostic implications of dysphagia in elderly patients with pneumonia. Age Ageing 2010;39:39-45.

9 Clavé P, de Kraa M, Arreola V, et al. The effect of bolus viscosity on swallowing function in neurogenic dysphagia. Aliment Pharmacol Ther 2006;24:1385-94.
10 Cook IJ, Kahrilas PJ. AGA technical review on management of oropharyngeal dysphagia. Gastroenterology 1999;116:455-78.

11 Altman KW, Yu G-P, Schaefer SD. Consequence of dysphagia in the hospitalized patient: impact on prognosis and hospital resources. Arch Otolaryngol Head Neck Surg 2010;136:784-9.

12 European Society for swallowing disorders (2012) ESSD position statements: oropharyngeal dysphagia in adult patients. Available: www.myessd.org/docs/position_statements/ESSD_Position_ Statements_on_OD_in_adult_patients_for_web.pdf [Accessed 10 Feb 2019].

13 Cabib C, Ortega O, Kumru H, et al. Neurorehabilitation strategies for poststroke oropharyngeal dysphagia: from compensation to the recovery of swallowing function. Ann N Y Acad Sci 2016;1380:121-38.

14 Ickenstein GW, Riecker A, Höhlig C, et al. Pneumonia and in-hospital mortality in the context of neurogenic oropharyngeal dysphagia (NOD) in stroke and a new NOD step-wise concept. J Neurol 2010;257:1492-9.

15 Rofes L, Arreola V, Almirall J, et al. Diagnosis and management of oropharyngeal dysphagia and its nutritional and respiratory complications in the elderly. Gastroenterol Res Pract 2011;2011:1-13.

16 Liberati A, Altman DG, Tetzlaff J, et al. The PRISMA statement for reporting systematic reviews and meta-analyses of studies that evaluate health care interventions: explanation and elaboration. BMJ 2009;21:b2700.

17 Marin S, Serra-Prat M, Ortega O, et al. Cost of oropharyngeal dysphagia after stroke: a systematic review. prospero: International prospective register of systematic reviews. 2018. CRD42018099977. Available: https://www.crd.york.ac.uk/prospero/display_record.php? RecordID=99977

18 Marin S, Serra-Prat M, Ortega O, et al. Cost of oropharyngeal dysphagia after stroke: protocol for a systematic review. BMJ Open 2018;8:e022775.

19 Centre for Reviews and Dissemination. Systematic Reviews: CRD's Guidance for Undertaking Reviews in Health Care. York: Centre for Reviews and Dissemination, University of York, 2009.

20 Drummond MF, O'Brien B, Torrance GW, et al. Methods for the economic evaluation of healthcare programmes. 2nd ed. Oxford: Oxford University Press, 1997.

21 Guyatt G, Oxman AD, Akl EA, et al. Grade guidelines: 1. IntroductionGRADE evidence profiles and summary of findings tables. J Clin Epidemiol 2011;64:383-94.

22 Wojner AW, Alexandrov AV. Predictors of tube feeding in acute stroke patients with dysphagia. AACN Clin Issues 2000;11:531-40.

23 Bonilha HS, Simpson AN, Ellis C, et al. The one-year attributable cost of post-stroke dysphagia. Dysphagia 2014;29:545-52.

24 Chen C-M, Chang C-H, Hsu H-C, et al. Factors predicting the total medical costs associated with first-ever ischeamic stroke patients transferred to the rehabilitation ward. J Rehabil Med 2015;47:120-5.

25 Chen C-M, Ke Y-L. Predictors for total medical costs for acute hemorrhagic stroke patients transferred to the rehabilitation ward at a regional hospital in Taiwan. Top Stroke Rehabil 2016;23:59-66.

26 Muehlemann N, Jouaneton B, de Léotoing L, et al. Hospital costs impact of post ischemic stroke dysphagia: database analyses of hospital discharges in France and Switzerland. PLoS One 2019;14:e0210313.

27 Katzan IL, Dawson NV, Thomas CL, et al. The cost of pneumonia after acute stroke. Neurology 2007;68:1938-43.

28 Christensen MC, Previgliano I, Capparelli FJ, et al. Acute treatment costs of intracerebral hemorrhage and ischemic stroke in Argentina. Acta Neurol Scand 2009;119:246-53.

29 Christensen MC, Valiente R, Sampaio Silva G, et al. Acute treatment costs of stroke in Brazil. Neuroepidemiology 2009;32:142-9.

30 Wilson RD. Mortality and cost of pneumonia after stroke for different risk groups. J Stroke Cerebrovasc Dis 2012;21:61-7.

31 Gomes F, Emery PW, Weekes CE. Risk of malnutrition is an independent predictor of mortality, length of hospital stay, and hospitalization costs in stroke patients. J Stroke Cerebrovasc Dis 2016;25:799-806.

32 Patel DA, Krishnaswami S, Steger E, et al. Economic and survival burden of dysphagia among inpatients in the United States. Dis Esophagus 2018;31:1-7.

33 Attrill S, White S, Murray J, et al. Impact of oropharyngeal dysphagia on healthcare cost and length of stay in hospital: a systematic review. BMC Health Serv Res 2018;18:594. 